\title{
Estudos em Sapindaceae I. Novas espécies de Talisia Aublet para o Brasil
}

\author{
Germano Guarim Neto $[*]$
}

\begin{abstract}
Resumo
O autor apresenta quatro novas espécies do gênero Talisia Aublet (Sapindaceae) para o Brasil: Talisia amazonica, Talisia medrii, Talisia prancei e Talisia veraluciana.
\end{abstract}

Talisia amazonica G. Guarim Neto, sp. nov. (Fig. 1)

Secção Talisia Subsecção Acladodea (R. \&

P.) Radlk.

Sufírutex vel arbuscula usque $6 \mathrm{~m}$. alta; ramis villosissimis ferrugineis. Folia paripinnata; petiolo $13-17 \mathrm{~cm}$. longo tereti striato, villosissimo cinereo, rachidi tereti striata villosissima cinereo, obscura; foliolis 2-4 jugis, $13-45 \mathrm{~cm}$. longis, $6-12 \mathrm{~cm}$. latis exacte oppositis vel alternis, oblongo-lanceolatis supra glabris vel nervis principalibus puberulis, subtus nervis principalibus lanatis et nervis secundariis terciariisque puberulis, utrinque fuscis, longe acuminatis, acumine usque ad $2,5 \mathrm{~cm}$. longo, basi inaequilateris; petiolulo $2-8 \mathrm{~mm}$. longo, crasso, villosissimo, subcoriaceo; nervis lateralibus utrinque ad 25 , nervis principalibus utrinque prominentibus, nervatione brochidroma, venatione forte reticulata. Inflorescentiae paniculatae, $9 \mathrm{~cm}$. Iongre $3 \mathrm{~cm}$. latae, terminales vei axillares, bracteatae, bracteis linearibus, villosis, rhachidibus et ramis floriferis villosissimis. Flores flavi $5 \mathrm{~mm}$. longi. pedicello $3 \mathrm{~mm}$. longo, bracteolato, bracteolis linearibus, viliosis. Calyx ad infra medium partitus, sepalis ovatis acutis carinatis carnosis extus puberulis, intus glabris, margine ciliatis, unguiculatis, ungue intus glabro. Petala calyce duplo longiora, oblonga, glabra, ad marginem ciliolata, unguiculata, ungue ad marginem ciliato. Squama simplix petalo aequans, extus glabra, intus villossissima, ligulata, ligula elongata, glabra. Discus annularis lobatus pentagonalis hirtellus. Stamina filiformia, parum villosa, antheris linea- ribus, oblongis, leviter falcatis, apiculatis. Ovarium immaturum, ovoideum, ad apicem dense setulosum. Fructus juvenis usque ad $2,5 \mathrm{~cm}$. Iongus, oblongus, granulatus, apiculatus, monospermus, calyce persistenti. Semina elongata, crassiuscula.

Tipos - W. A. Rodrigues 8989. BRASIL. Amazonas : Estrada Manaus-Itacoatia$\mathrm{ra}, \mathrm{Km} 155$, picada XIX, a $400 \mathrm{~m}$ da estrada, fl, 4/11/1970 (holótipo INPA). B. W. Albuquerque et al. 500. Lago de Coari, Município de Coari, fr, 23/2/1972 (parátipo INPA). L. Coelho 132. Estrada Manaus-Porto Velho, Km 85, fr, 20/12/1971 (parátipo INPA). N. T. Silva 4380 . Tefé, Base do Projejeto RADAM, estrada que vai para a Colônia, fr, 16/8/1976 (parátipo IAN).

J. G. Kuhlmann 1166. Codajás, rio Solimões, fr, 19/1/1924 (parátipo RB). J. M. Pires 3968. Pará, Alto Tapajós, Vila Nova, perto da cachoeira do Chacorão, fr, 19/1/1952 (parátipo IAN).

NoMe vUlgar - BRASIL. Amazonas: Pitomba (ex J. G. Kuhlmann 1166).

Fenologia - Floresce em novembro e trutifica de dezembro a janeiro, ocasional. mente em agosto.

HABITAT E DistribuiÇão - Encontrada preferentemente nas matas de terra firme e distribuida pelos Estados do Amazo. nas e Pará.

$T$. amazonica assemelha-se muito à $T$. macrophylla e dela se afasta principalmente pelo tamanho e disposição dos folíolos, pelas brácteas da inflorescência, na pilosidade dos ramos. pecíolo e peciólulo, assim como nos frutos e sementes.

$T$. amazonica é uma homenagem à rica região amazônica da qual foi retirada a maioria das coleções para o estudo posterior do gênero no Brasil.

(") - Fundação Universidade Federal de Mato Grosso, Cuiabá. Bolsista CAPES. Curso de Pós-Graduaçăo do INPA/FUA, Manaus. 


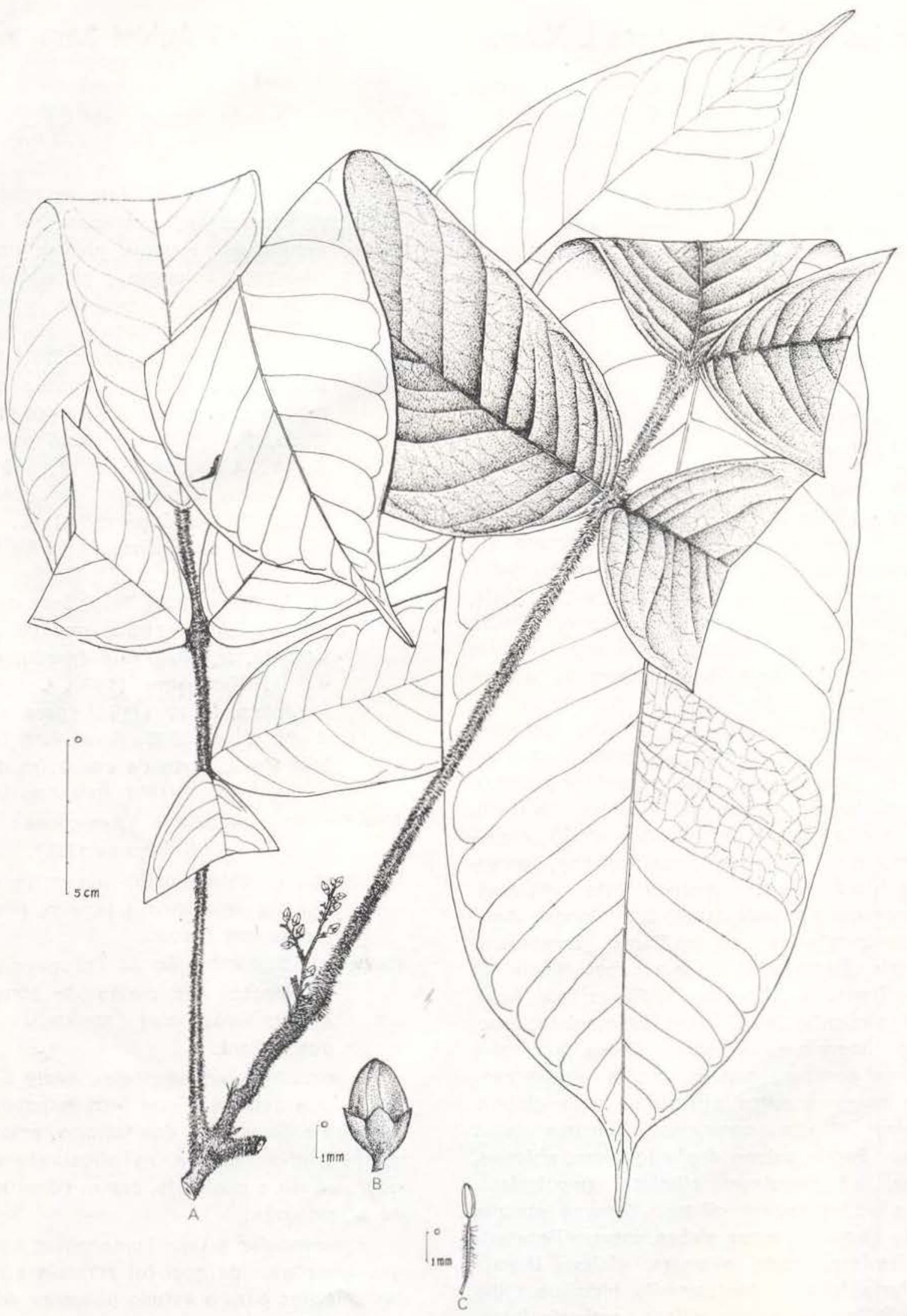

Fig. 1. Talisia amazônica G. Guarim Neto, sp. nov.: A. Ramo florifero; B. Botão floral; C. Estame. (Desenho de A. Silva). 
Talisia medrii G. Guarim Neto, sp. nov. (Fig. 2)

Secçāo Talisia Subsecção Acladodea (R. \& P.) Radlk.

Frutex vel arbuscula; ramis teretibus hirtellis punctatis. Folia pari-(impari-) pinnata, petioio $8-20 \mathrm{~cm}$. longo, tereti, striato, hirtello, rhachidi subtrigona, hirtella; foliolis $3-4$ jugis oppositis vel alternis, oblongis $8-21 \mathrm{~cm}$. longis, $2,5-6 \mathrm{~cm}$. latis, breviter acuminatis (acumine usque ad $2 \mathrm{~cm}$. longo) basi cuneatis, inaequilateris, supra glaberrimis subtus ad vel vix supra medium nervi principalis cum pilis munitis, utrinque opacis; petiolulo $7 \mathrm{~mm}$. longo crasso verruculoso dense hirtello subcoriaceo; nervis lateralibus utrinque usque 15 , nervis principalibus supra vix prominentibus, subtus valde prominentibus, nervatione brochidodroma, venatione valde reticulata. Inflorescentiae paniculatae, $30 \mathrm{~cm}$. usque longae, $25 \mathrm{~cm}$. latae, terminales, pyramidales, ramosae, bracteatae. rhachidibus et ramis floriferis striatis dense hirteilis, dichasium congestum gerentibus. Flores albi, $8 \mathrm{~mm}$. usque longi, pedicello $3 \mathrm{~mm}$ usque longo bracteolato, toto ciliolato. Calyx ad medium usque partitus, sepalis ovatis carinatis extus ciliolatis, intus glabris, ad marginem dense ciliatis. Petala calyce duplo longiora, oblonga, extus ad basin villosa intus parum pilosula et glandulis conspersa, ad marginem parum ciliata, unguiculata, ungue extus pilosulo intus glabrato. Squama petalo aequilonga, simplex, extus villosa intus villosissima, ligulata, ligula extus glabra et ad marginem ciliata, intus tantum ultra medium pilosula. Discus angulatus crenatus, ad apicem et angulos setulosus. Stamina filiformia leviter complanata, superne villosa, glabrescentia, antheris linearibus cordatis falcatis apiculatis. Ovarium rudimentarium vel immaturum ovoideum dense setulosum, triloculare, utroque loculo uniovulato, ovulorum placentatione parietali, stylo setuloso, stigmate semipapilloso. Fructus juvenis usque $2,5 \mathrm{~cm}$. longus, elongatus trisulcatus granulatus vel apiculatus, puberulentus, calyce persistenti subtentus, maturus $3 \mathrm{~cm}$. longus elongatus trisulcatus longitudine granulatus, apiculatus, puberulentus, calycis reliquiis saepissime subtentus, monospermus Semen nitidum arillatum, testa verrucosa, co tyledonibus crassiusculis.
Trpos - M. E. Medri 33. BRASIL. Amazonas, rio Negro, Anavilhanas, fl, 3/3/1976 (holótipo INPA). J. G. Kuhlmann 206. Manaus, fl, dezembro de 1913 (parátipo RB). P. B. Cavalcante 3268. Rio Negro, Anavilhanas, rio Ariaú, fl, 29-2-1976 (parátipo INPA). M. F. Silva et al. 1904. Paraná do Sumaúma, acima de Anavilhanas, fr, 2/2/1976 (parátipo INPA). G. Guarim Neto 16. id., $\mathrm{fl} \& \mathrm{fr}$, 28/2/1976 (parátipo INPA).

NOME VULGAR - BRASIL - Amazonas : Lacrebranco (ex J. G. Kuhlmann 206).

Fenologia - Floresce de dezembro a março e frutifica de fevereiro a março.

HABITAT E DISTRIBUIÇÃo - Habita a mata periodicamente inundada (igapó), de solo argilo-arenoso. Encontrada no Estado do Amazonas, especialmente no arquipélago de Anavilhanas, rio Negro.

$T$. medrii tem afinidades com $T$. cerasina, da qual difere principalmente pelo tamanho da flor, forma das pétalas e escamas, tamanho e forma da inflorescência e dos frutos e é preferentemente de igapó, enquanto $T$. cerasina ocorre em diferentes habitats.

T. medrii é dedicada ao coletor de sua amostra padrão, Moacyr E. Medri, um bom amigo e excelente anatomista.

Talisia prancei G. Guarim Neto, sp. nov . (Fig. 3)

\section{Secção Talisia Subsecção Pitombaria Radlk.}

Frutex vel arbor $10 \mathrm{~m}$. alta, $15 \mathrm{~cm}$. diametro; ramis cylindricis striatis glabris lenticellatis. Folia impari-(pari-) pinnata; petiolo $2-4 \mathrm{~cm}$. longo cylindrico striato dense puberulo glabrescenti, rhachidi parum anguloso dense puberula, foliolis $2-5$ jugis usque ad $10 \mathrm{~cm}$. longis usque ad $4 \mathrm{~cm}$. latis, alternis oppositis ovato-ellipticis supra glabris vel nervis principalibus cum pilis paucis munitis subtus sparse setulosis basi inaequilateris apice obtusis peticlulo circa $0,5 \mathrm{~cm}$. longo semicylindrico hirtello, subcocriaceo; nervis lateralibus utrinque circa 11, nervis principalibus supra vix prominentibus infra prominentibus, nervatione 


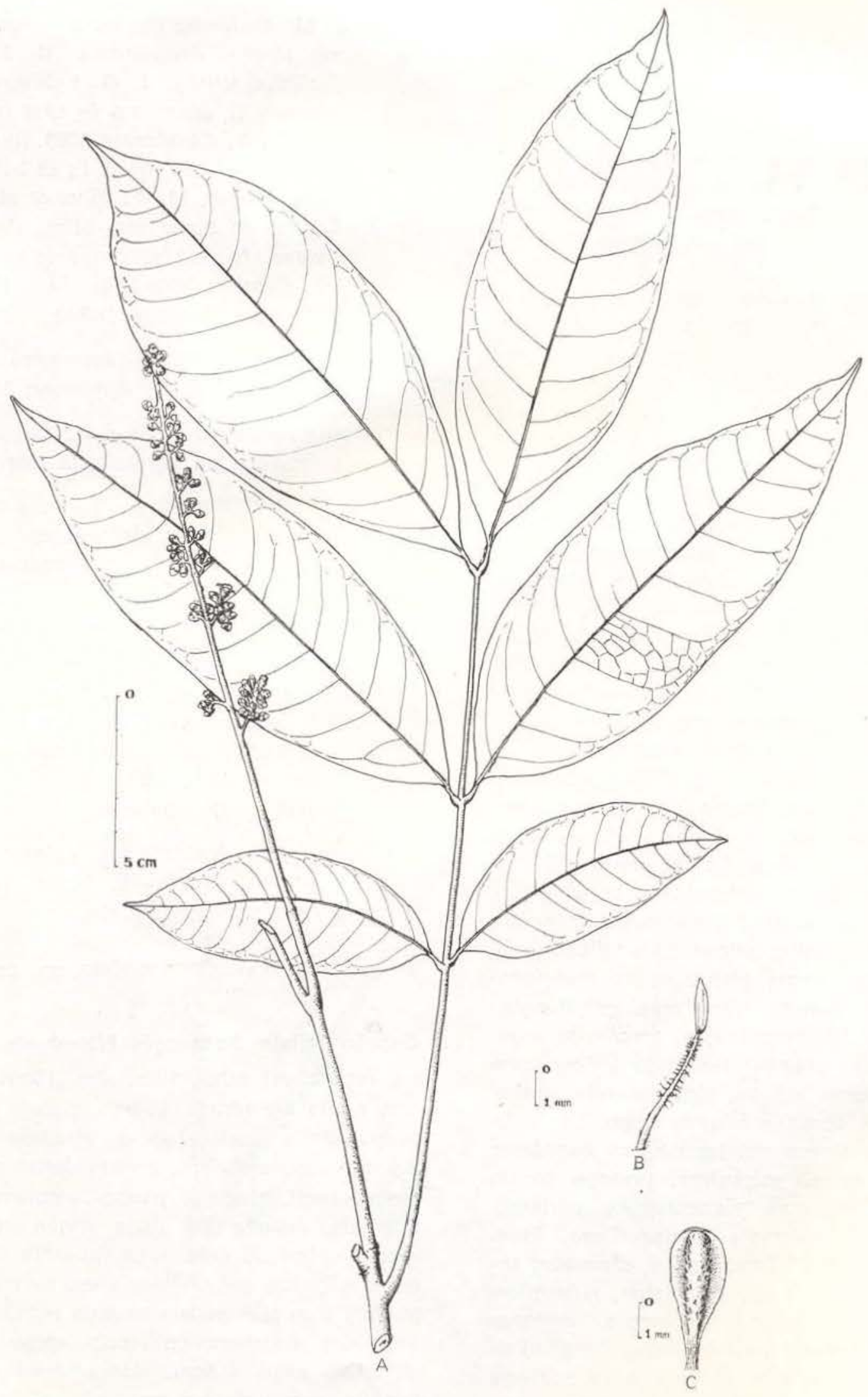

Fig. 2. Talisia medrii G. Guarim Neto, sp. nov.: A. Ramo florífero; B. Estame; C. Pétala com escama, (Desenho de J. Palheta). 
brochidodroma, eucamptodroma, venatione den se reticulata. Inflorescentiae paniculatae, circa $20 \mathrm{~cm}$. longae, circa $8 \mathrm{~cm}$. latae, terminales vel subterminales, bracteatae, bracteis dense villosis, rachidibus floriferis angulosis, hirtellis; dichasiis paucifloris gerentibus. Fiores viriduli circa $3-4 \mathrm{~mm}$. longi; pedicelli circa $2 \mathrm{~mm}$. longi, hirsuti, bracteolati, bracteolis vil!osis. Calyx fere ad basin partitus, sepalis obtusis extus dense hirtellis, intus glabrescentia vel puberulis, marginibus ciliatis. Petala calyce fere duplo longiora, oblonga, acuta, extus puberula vel

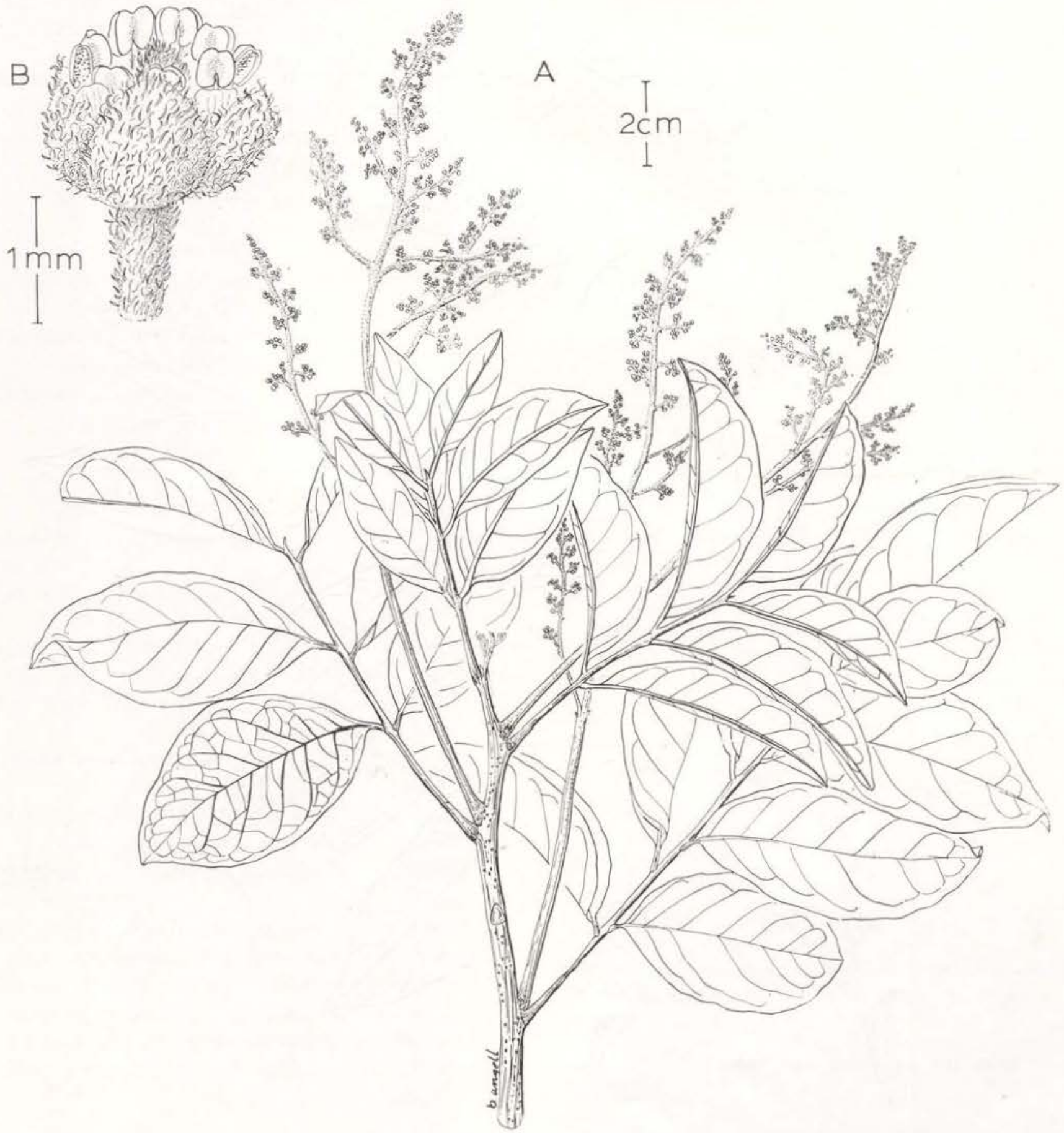

Fig. 3. Talisia prancei G. Guarim Neto, sp. nov.: A. Ramo florifero; B. Botāo floral quase aberto, mostrando as anteras (Desenho de B. Angell). 


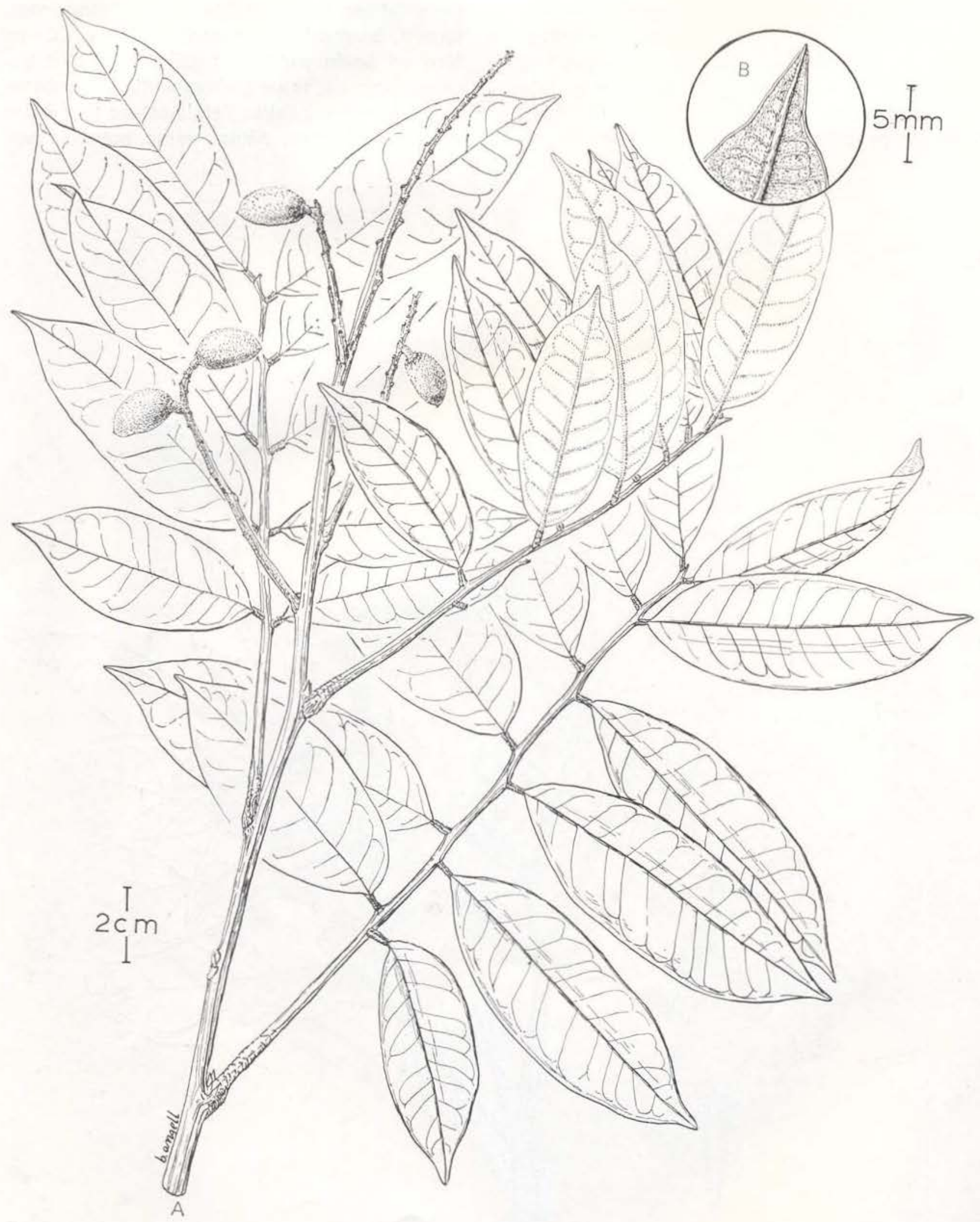

Fig. 4. Talisia veraluciana G. Guarim Neto, sp. nov.: A. Ramo frutífero; B. Aspecto da face inferior do foliolo. (Desenho de B. Angell). 
glabrescentes, intus parum villosa, ungue indistincto. Squamae villosissimae; ligula absenti. Discus crenatus glabrus. Stamina filiformia, basi leviter inflata, vix supra medium villosa, antheris oblongis cordatis. Ovarium ovoideum dense setulosum, stigmate setuloso. Fructus juvenis usque ad $0,5 \mathrm{~cm}$. Iongus ovoideus apiculatus dense puberulentus, calyce persistenti.

TIPOS - G. T. Prance et al. 59651. BRASIL. Goiás. $40 \mathrm{~km}$ de Caiapônia, fl, 25/10/1964 (holótipo NY; isótipos F; GH; MO; S; US). W. Archer et al. 176. Mato Grosso (do Sui), Campo Grande, $\mathrm{fl} \& \mathrm{fr}$ jov, 10/9/1936 (parátipo US).

G. T. Prance et al. 59182. Mato Grosso, serra do Roncador, fl, 30/9/1964 (parátipos F; GH; NV; S; UC; US).

Fenologia - Coletada com flor e frutos jovens em setembro e outubro.

HABTTAT E DisTrIBUIÇÃo - Característica dos cerrados dos Estados de Goiás, Mato Grosso e o atual Mato Grosso do Sul.

T. prancei assemelha-se à $T$. retusa, da qual se afasta principalmente pelo tamanho e forma dos folíolos, tamanho e coloração das flores e pela pilosidade dos frutos jovens.

$T$. prancei é dedicada ao seu principal coletor, G. T. Prance, que ofereceu facilidades para a continuação dos nossos estudos em The New York Botanical Garden (USA).

Talisia veraluciana G. Guarim Neto, sp. nov. (Fig. 4)

Secção Talisia Subsecção Pitombaria Radlk.

Arbor circa $27 \mathrm{~m}$. alta; ramis cylindricis sulcatis verrucosis pulverulentis lenticellatis. Folia pari-(impari-) pinnata, petiolo $4-7,5 \mathrm{~cm}$. longo forte striato puberulento, gemmis axillaribus tomentosis, rhachide subtriangulari puberula; foliolis 4-6 jugis, alternis vel oppositis, oblongis acuminatis $3-10 \mathrm{~cm}$. longis, $4-10 \mathrm{~cm}$. usque latis, coriaceis utrinque fuscis, supra praeter costam glabris, infra nervis principali- bus dense aureo-setulosis; petiolulo $0,5 \mathrm{~cm}$. usque longo angulato verruculoso puberulento setuloso, nervis lateralibus utrinque usque ad 15. nervis principalibus supra alte impressis infra forte prominulis, nervatione brochidodro. $\mathrm{ma}$, venatione reticulata. Fructus in paniculas terminales longas pyramidales dispositi, rhachidi striata pulverulenta vel minutim setulosa. Fructus juvenis $1,8 \mathrm{~cm}$. longus ovoideus vel oblongus apiculatus dense puberulentus, calycis reliquiis basin usque partitus subtentus, sepaiis ovatis acutis; monospermus. Semen junius nigrescens.

Tipos - B. A. Krukoff 6886. BRASIL. Amazonas, Município de Humaitá, fr, 1934 (holótipos BR; isótipos A; BM; F; MO)

Fenologia - Coletada com frutos em outubro e novembro.

HABITAT E DISTRIBUiçÄO - Encontrada nas matas de terra firme do Estado do Amazonas.

$T$. veraluciana tem afinidades com $T$. clathrata e dela se afásta principalmente pelo número de foliolos, tamanho do pecíolo, pela pilosidade dos frutos e na coloração dourada da face inferior dos folíolos.

$T$. veraluciana é dedicada à minha esposa Vera Lucia, companheira sempre incentivadora.

\section{Agradecimentos}

Aos Drs. William A. Rodrigues, Ghillean T. Prance e Rupert Barneby pelas correções latinas das espécies.

\section{SUMMARY}

The author describes in this paper four new species of the genus Talisia Aublet (Sapindaceae) from Brazil: Talisia amazonica, Talisia medrii, Talisia prancei and Talisia veraluciana.

(Aceito para publicação em 20/02/79) 